\title{
Gene expression profile of Scardovia spp. in the metatranscriptome of root caries
}

\section{Naile DAMÉ-TEIXEIRA ${ }^{(a)}$ \\ Clarissa Cavalcanti Fatturi PAROLO(b) \\ Marisa MALZ(b) \\ Deirdre Ann DEVINE(c) \\ Thuy DO(c) (iD)}

(a) Universidade de Brasília - UnB, Faculty of Heath Sciences, Department of Dentistry, Brasília, DF, Brazil.

(b) Univeridade Federal do Rio Grande do Sul - UFRGS, Faculty of Dentistry, Department of Social and Preventive Dentistry, Porto Alegre, RS, Brazil.

(c) University of Leeds, School of Dentistry, Division of Oral Biology, Leeds, England.

Declaration of Interests: The authors certify that they have no commercial or associative interest that represents a conflict of interest in connection with the manuscript.

\section{Corresponding Author:}

Nailê Damé-Teixeira

E-mail: nailedame@hotmail.com

hitps://doi.org/10.1590/1807-3107bor-2020.vol34.0042

Submitted: November 19, 2019

Accepted for publication: March 16, 2020

Last revision: April 9, 2020
Abstract: A few investigations of caries biofilms have identified Scardovia spp.; however, little is known about its involvement in caries pathogenesis. The purpose of this study was to assess the gene expression profile of Scardovia spp. in root caries, and compare it with other microorganisms. Clinical samples from active root caries lesions were collected. Microbial mRNA was isolated and cDNA sequenced. The function and composition of the Scardovia were investigated using two methods: a) de novo assembly of the read data and mapping to contigs, and b) reads mapping to reference genomes. Pearson correlation was performed ( $\mathrm{p}<0.05)$. Proportion of Scardovia inopinata and Scardovia wiggsiae sequences ranged from $0-6 \%$ in the root caries metatranscriptome. There was a positive correlation between the transcriptome of Lactobacillus spp. and Scardovia spp. $(\mathrm{r}=0.70 ; \mathrm{p}=0.03)$, as well as with other Bifidobacteriaceae $(r=0.91 ; p=0.0006)$. Genes that code for fructose 6-phosphate phosphoketolase (the key enzyme for "Bifid shunt"), as well as ABC transporters and glycosyl-hydrolases were highly expressed. In conclusion, "Bifid shunt" and starch metabolism are involved in carbohydrate metabolism of S. inopinata and $S$. wiggsiae in root caries. There is a positive correlation between the metabolism abundance of Lactobacillus spp., Bifidobacteriaceae members, and Scardovia in root caries.

Keywords: Root Caries; High-Throughput Nucleotide Sequencing; RNA; Biofilms.

\section{Introduction}

Dental biofilms are microbial communities with a complex structure, in which each organism has specific requirements for growth and survival. Consequently, many species, metabolic pathways for utilisation of different nutrients and cell protection against stress factors are found in a biofilm. ${ }^{1}$ This diversity of species and metabolism conserves the homeostasis within the biofilm, ${ }^{2}$ which can be disrupted by an environmental change that leads to progression to caries. ${ }^{3}$ Many species have been found in dental caries biofilms ${ }^{4}$ but studies still focus on well-established cariogenic microorganisms, Streptococcus mutans and Lactobacillus spp. S. mutans is well-documented as an important (but not necessary) organism for caries. Lactobacillus spp. are associated with root caries progression, but were absent or rarely observed in individuals without root caries, ${ }^{5}$ 
in accordance with the concept that they are weakly adherent and depend on ideal niches with retention to be able to colonize. ${ }^{6}$

Some studies have unveiled a massive prevalence of the Bifidobacteriaceae family in both coronal ${ }^{7,8}$ and root caries. ${ }^{9,10}$ Similarly to lactobacilli, the Bifidobacteriaceae family has no ability to form biofilms by itself, ${ }^{11}$ requiring a retentive niche to colonize. Some species of this family have been consistently found in root caries lesions, and amongst them, Scardovia spp. has been identified as a member of caries lesion biofilms, especially in severe early childhood caries. ${ }^{12,13,14}$ Scardovia spp. was one of the members of the oral microbiota with low abundance $(<0.01 \%)$ that significantly influenced the microbial community structure and could be associated with disruption of homeostasis. ${ }^{15}$ Prevalence of this genus was associated with root caries activity., ${ }^{9,10,16}$

It is still unknown whether Scardovia spp. metabolism may play a major role in dental caries biofilms. Their cariogenic potential was investigated in a few studies ${ }^{11,17}$ and their acid production was shown to be higher when in association with $S$. mutans in vitro. ${ }^{11}$ As heterofermentative organisms, Scardovia spp. can metabolize several carbohydrates with lactate and acetate as major end products of fermentation. ${ }^{18}$ These products could help in the biofilm acidification leading to dysbiosis. The purpose of this study was to explore the gene expression profiles of Scardovia inopinata and Scardovia wiggsiae in root caries, and compare it with that of Streptococcus mutans, Lactobacillus spp., which are well-established cariogenic microorganisms, and other Bifidobacteraceae members.

\section{Methodology}

\section{Clinical collection and experimental approach}

Volunteers to the study were patients who attended the dental clinics for any dental treatment in two centres: a) Faculty of Dentistry, Federal University of Rio Grande do Sul, Porto Alegre, Brazil; and b) Leeds School of Dentistry, University of Leeds, Leeds, United Kingdom. This study was approved by the ethics committee of the Federal University of Rio Grande do Sul (process no 427.168) and by the ethics committee of the NRES Committee Yorkshire \& The Humber - Leeds West (protocol n ${ }^{\circ}$ 2012002DD). Ethical approval was obtained for the samples collection and all volunteers signed a formal consent.

Details of the clinical collection and experimental approach are described elsewhere. ${ }^{19}$ Briefly, clinical samples (biofilm + carious dentin) were collected from volunteers $(\mathrm{n}=30)$ presenting an active cavitated root caries lesion. The diagnosis of root caries was done according to the activity criteria, using visual-tactile examination. ${ }^{20}$ Excavated carious dentin containing soft and infected tissue was collected using a sterile spoon excavator (SSWhiteDuflex, Rio de Janeiro, Brazil). The mean age of the volunteers who donated root caries samples was $60.1 \pm 11.6$ years (range 40-90 years).

After collection, samples were immediately placed in a reagent for RNA stabilization (Qiagen Inc.. Manchester, UK). The total RNA was extracted using the UltraClean ${ }^{\circledR}$ Microbial RNA Isolation kit (Mo-bio, San Diego, USA) with on-column DNAse digestion (Qiagen Inc., Manchester, UK). Samples with a total RNA concentration < 30ng/RNA were pooled, leading to a final sample of seven pools and two unique samples, with good mRNA quantity and quality. The total RNA was depleted of ribosomal RNA using the Ribo-Zero ${ }^{\mathrm{TM}}$ Meta-Bacteria Kit (Epicentre, Illumina, San Diego, USA). Illumina ${ }^{\circledR T r u S e q}{ }^{\mathrm{TM}}$ library prep protocols (Illumina, San Diego, USA) were used for library preparation from the enriched mRNA. Sequencing was performed on the Illumina HiSeq2500 (Illumina Inc., San Diego, USA) sequencer to obtain $2 \times 100 \mathrm{bp}$ sequence reads.

\section{Bioinformatics analysis}

RNA sequencing data are available from the National Center for Biotechnology Information (NCBI) Sequence Read Archive, under the accession number SRS779973. Two different pipelines were used to carry out the bioinformatics analysis, as described below.

\section{Pipeline a) Annotation of de novo assembled contigs}

Read data were obtained as FASTQ files and were quality trimmed using cutadapt (https:// 
github.com/marcelm/cutadapt). The taxonomic profiling was carried out by denovo assembly of the reads into contigs using the default parameters at CLC Genomics Workbench 7.5.1 software (CLC Bio, Qiagen, Manchester, UK). Species and gene annotation of the contigs was carried out using diamond (https://ab.inf.uni-tuebingen.de/software/ diamond/) with the NCBI nr protein database (downloaded in December 2017), with settings of $70 \%$ similarity to the reference database. Reads from each sample were then mapped against the contigs using the CLC Genomics Workbench 7.5.1 software and a read count table was obtained.

The number of reads assigned to Scardovia spp., Bifidobacteriaceae members, Lactobacillus spp. and $S$. mutans in the total metatranscriptome was assessed and plotted (RStudio for Mac, version 1.1.463, Plotly $\mathrm{R}$ package, ggplot2 $\mathrm{R}$ package). The proportion of species in the total metatranscriptome was calculated as follows: [sum of unique reads by species or genera / total number of mapped reads $\left.{ }^{*} 100\right]$. This was considered as the relative abundance of each species/genus in each sample. Pearson correlation was performed to evaluate the degree of correlation (and the direction of correlation - whether positive or negative) between the appraised organisms (RStudio, base R package; $p<0.05)$, after checking the normality of the data with the Shapiro-Wilk test.

\section{Pipeline b) Read mapping to 162 oral bacterial genomes}

Scardovia spp. gene expression was analysed by mapping reads to 162 oral bacterial reference genomes, including S. inopinata JCM 12537 and S. wiggsiae F0424. Quality-trimmed FASTQ files for each sample were imported into the CLC Genomics Workbench 7.5.1 software (CLC Bio, Qiagen, Manchester, UK). Genomes and their associated information were downloaded from the DNA Data Bank of Japan, NCBI, the Broad Institute and HOMD database, and were used as reference genomes for short read mapping data and a count table was obtained. Then, the putative presence of Scardovia spp. in each sample was estimated by the following method: [sum of reads/total number of genes $\geq 1$ ]. Based on this calculation, one sample was excluded from this analysis due to the low number of reads mapped to Scardovia. The correlation between samples gene expression was plotted (RStudio for Mac, version 1.1.463, Plotly $\mathrm{R}$ package, ggplot2 $\mathrm{R}$ package, corrplot $\mathrm{R}$ package). Gene expression levels were normalized by dividing the number of reads mapping to a single gene from a bacterium by the total reads assigned to that corresponding bacterium and presented as percentages. In order to compare the gene expression of specific genes related to carbohydrate metabolism, differences in means of expression level for particular genes were tested (Wilcoxon Rank Sum Test, corresponding to the Mann-Whitney U test; RStudio, base R package; $p<0.05)$ after checking the distribution of the data (Shapiro-Wilk and Kolmogorov-Smirnov tests). The number of reads assigned to Scardovia spp., Bifodobacterium spp., Lactobacillus spp. and S. mutans in the total metatranscriptome of root caries was assessed and plotted (RStudio for Mac, version 1.1.463, Plotly R package).

\section{Results}

Pipeline a) Composition of the metabolically active microbiota and comparison with established cariogenic species and other Bifidobacteriaceae members

Figures 1 and 2 shows descriptive analyses of the number of reads in each sample in the genomes of S. wiggsiae and S. inopinata (reads of both species were summed and presented as Scardovia spp.), as well as species/genera conventionally recognized as cariogenic pathogens. The analyses showed a prevalence of reads assigned to $S$. inopinata and $S$. wiggisae ranging from 0 to $6 \%$ in all metatrascriptomes of root caries. Two samples presented no gene expression of S. inopinata at all and only $0.04-0.07 \%$ of $S$. wiggsiae. What stands out from these charts is the very high but variable gene expression associated with Lactobacillus spp. This group represented $0.4 \%$ to $50.3 \%$ of all the metatranscriptome, and included the sum of reads of Lactobacillus sp.; L. acidophilus; $L$. amylovorus; L. brevis; L. buchneri; L. casei; L. crispatus; L. delbrueckii; L. fermentum; L. gasseri; L. gastricus; L. helveticus; L. jensenii; L. johnsonii; L. paracasei; L. 
reuteri; L. rhamnosus; L. salivarius; amongst others. Other members of the Bifidobacteriaceae family gene expression in root caries ranged from $0.4 \%$ to $8.4 \%$ of the total metatranscriptome. This group included reads of Bifidobacterium sp., B. dentium, B. brevis, $B$. longum, B. termophilum and Parascardovia denticolens. S. mutans was very active in some samples $(19.32 \%$ of all metatranscriptome in RC_7, for example). The most striking result to emerge from the data is that samples with $S$. inopinata gene expression representing $>5 \%$ of the total microbial metatranscriptome tended to present lower expression of $S$. mutans, but higher expression of Lactobacillus sp. genes (RC_A, RC_B, RC_H).

The correlation between the proportion of Scardovia spp. and other cariogenic species in the total metatranscriptome was tested using Pearson's correlation after checking data normality. A consistent positive correlation between Scardovia spp. and Lactobacillus spp. was observed $(\mathrm{r}=0.70$; $\mathrm{p}=0.03$ ), as well as a positive correlation with other Bifidobacteriaceae members $(r=0.91 ; p=0.0006)$. In contrast, there was a non-significant negative weak correlation of Scardovia spp. and S. mutans $(\mathrm{r}=-0.34$; $\mathrm{p}=0.35)$ (Figure 3).

\section{Pipeline b) Gene expression analysis}

Considering in this analysis the cut-off of the putative presence of Scardovia spp. assigned to each sample was the number of reads/number of genes $\geq 1$, only one library had no expression of $S$. inopinata or S. wiggsiae (RC_E; a pool of samples of two lesions from two patients, originated from center 2). In this sample, high gene expression of Lactobacillus spp. and $S$. mutans was observed. One sample showed no expression of $S$. wiggsiae (RC_8; non-pooled sample, from a single patient, originated from centre 1); this was the sample with higher predominance of
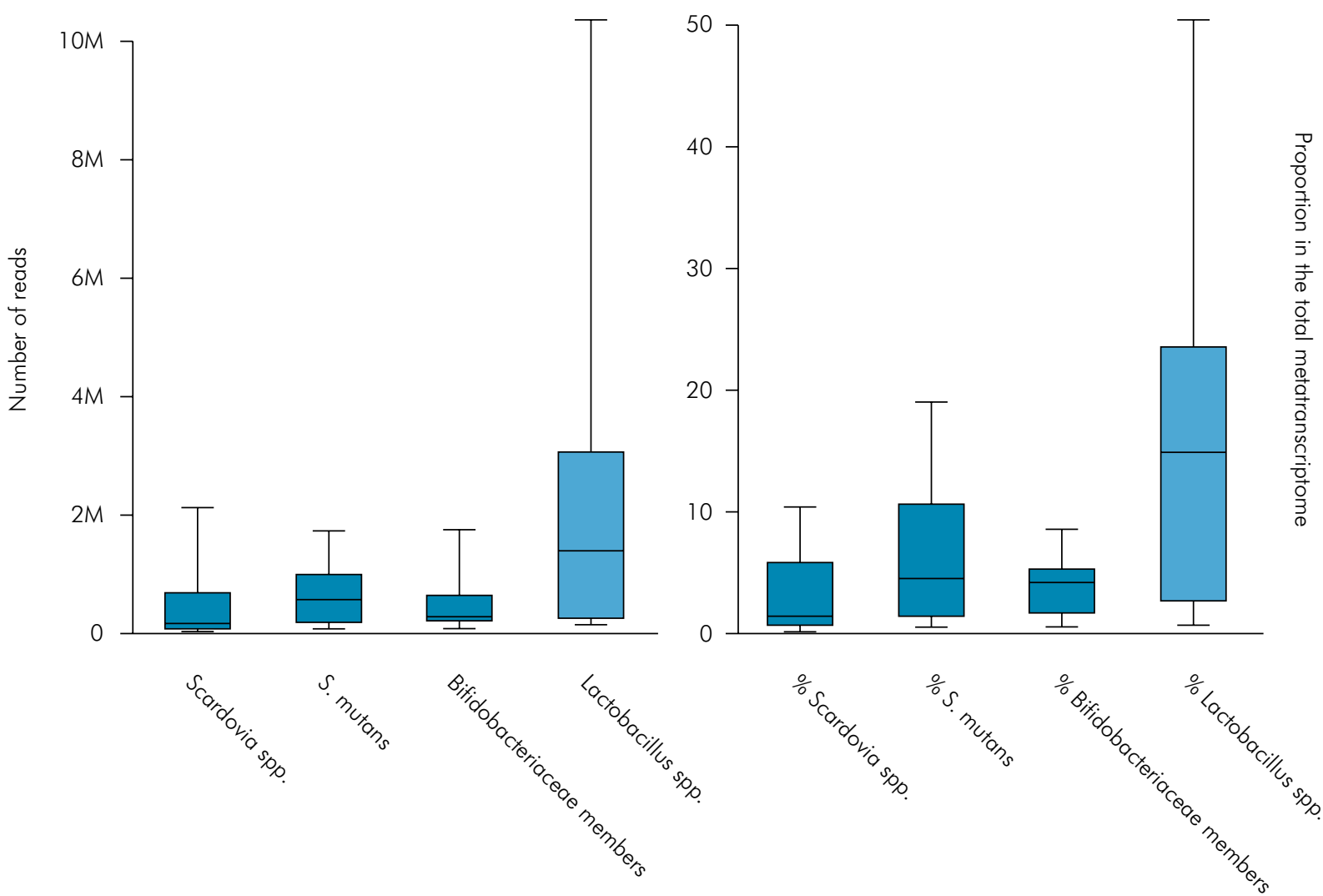

Figure 1. Number of reads and percentage in the total metatranscriptome of root caries biofilms of Scardovia spp., Bifidobacteriaceae members, Lactobacillus spp. and Streptococcus mutans (pipeline a). 


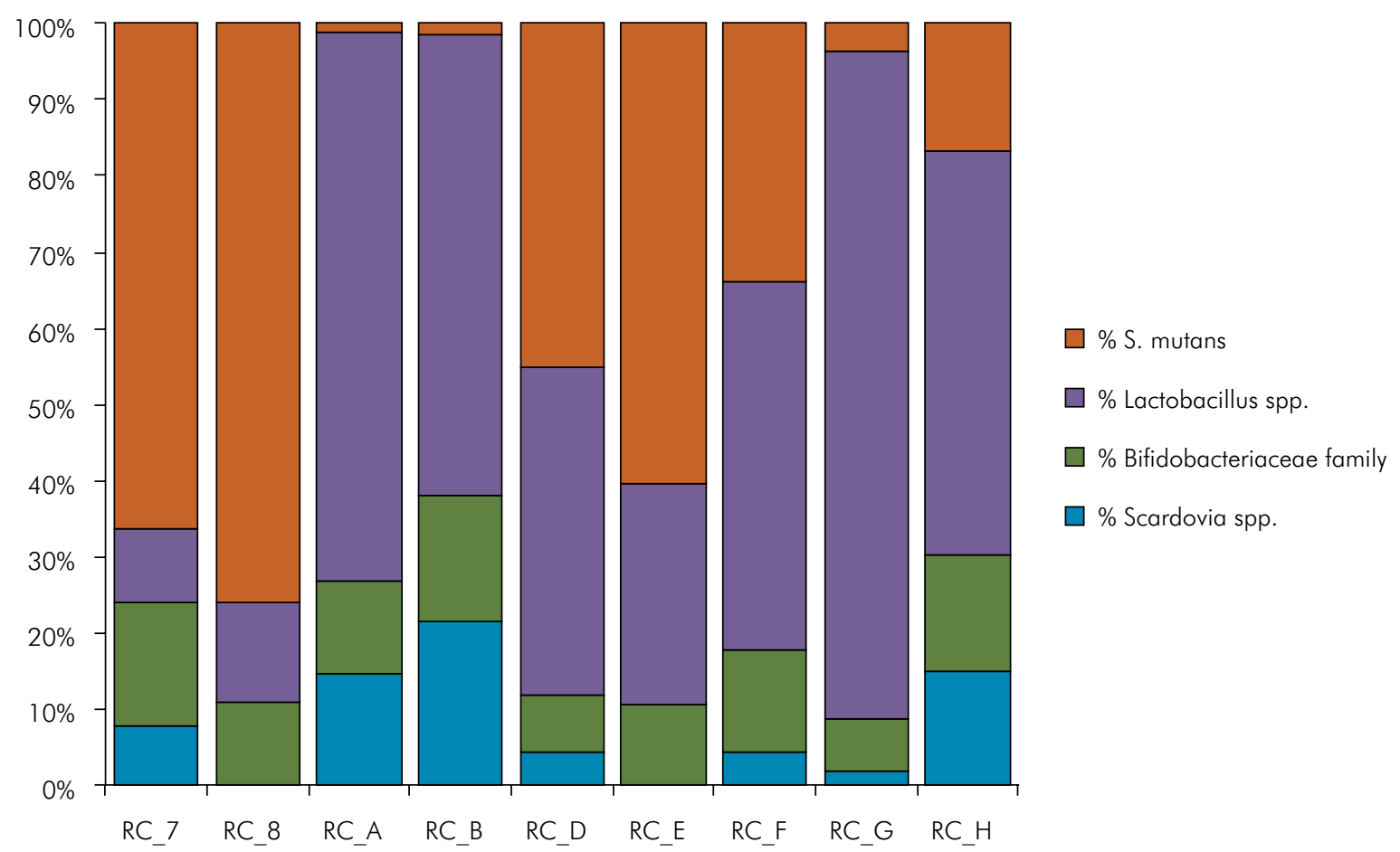

Figure 2. Proportion of reads of Scardovia spp., Bifidobacteriaceae members, Lactobacillus spp. and Streptococcus mutans in the metatranscriptome of root caries biofilms (pipeline a).
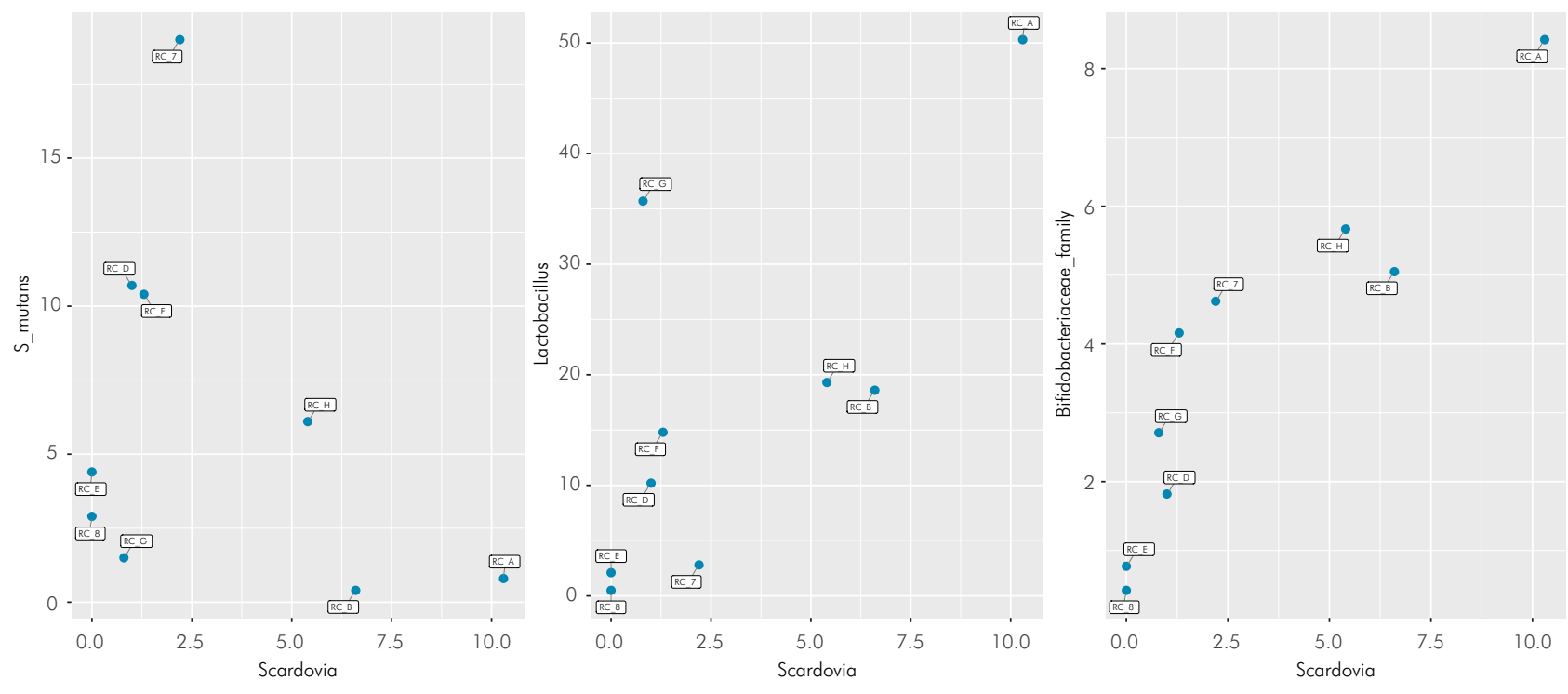

Figure 3. Correlation analysis of relative prevalence (\% in the total metatranscriptome) of Scardovia and other cariogenic species. (pipeline a). A: Streptococcus mutans proportion within the total metatranscriptome; B: Lactobacillus species proportion within the total metatranscriptome; C: Bifidobacteriaceae family proportion within the total metatranscriptome. Each point represents each sample (RC: Root Caries).

S. mutans in the analysed species. These samples were excluded from the gene expression analysis.
Figure 4 shows the correlation matrix between samples with putative presence of $S$. inopinata (A) and S. wiggsiae (B) from the number of reads of all genes 
- Gene expression profile of Scardovia spp. in the metatranscriptome of root caries
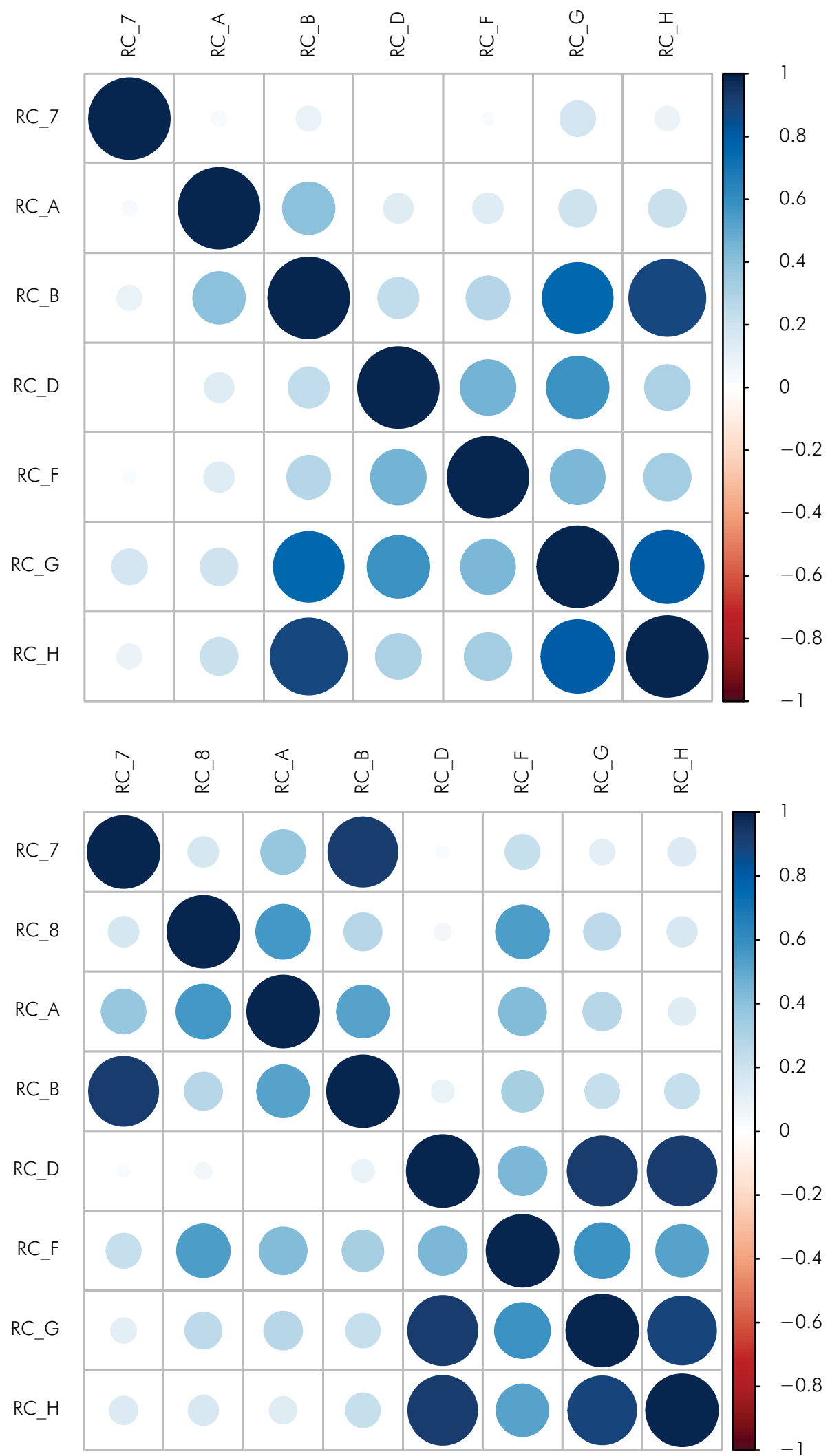

Figure 4. Degree of correlation analysis showing similarity of gene expression (number of reads) between all samples with putative presence of Scardovia spp. (A: S. inopinata; $n=8 ; B$ : S. wiggsiae; $n=7$ ). 
assigned to each species. From the chart, it can be seen that by far the greatest difference for both species was in the RC_D sample. This sample presented very low gene expression of Scardovia. Similarities between RC_7 and RC_B at S. wiggsiae could be observed, that was not observed for $S$. wiggsiae.

The Table shows the top 30 most expressed genes in both species. The most expressed gene represented a median of $2.2 \%$ of the transcription assigned to $S$. inopinata, that presented 170 genes with median of expression $>0.1 \%$. While for S. wiggsiae, the most expressed gene represented a median of $0.68 \%$ of the transcription and 264 genes had median of expression $>0.1 \%$, suggesting that the $S$. wiggsiae have a more complex set of functions. However, it could be observed that both species shared the majority of the highest expressed genes.

Gene expression analysis suggests the utilization of the "Bifid shunt" (oxidative pentose-phosphate (OPP) pathway) by S. inopinata and S. wiggsiae in root caries. There were differences in the ratios of expression of glucose-6-phosphate 1-dehydrogenase (median $=0.06 \%$, 25 th $-75^{\text {th }}=0.0 \%-0.1 \%$ ), and phosphoketolase (fructose 6-phosphate; median $=0.83 \% ; 25$ th $-75^{\text {th }}=0.2 \%-1.3 \%$ ) (Wilcoxon test, $\mathrm{p}=0.000$ ). Looking at the pentose phosphate pathway enzymes (Figure 5), it appears that phosphoketolase (EC 4.1.2.2) was the most expressed enzyme in both, S. inopitata and S. wiggsiae.

Another interesting aspect of the data is the expression of genes involved in lactic acid production which were highly expressed in S. inopinata and $S$. wiggsiae. Formate acetyltransferase ( $p f l)$ was one of the most expressed genes by Scardovia spp. in root caries and it was expressed in all samples. The $\mathrm{pfl}$ and lactate dehydrogenase (ldh) genes were highly expressed by S. inopinata ( $p f l$ median $=0.5 \% ; 25$ th $-75^{\text {th }}=0.2-0.8 \%$ and $l d h$ median $=0.12 \% ; 25$ th $-75^{\text {th }}=0-0.2 \%$ ). S. wiggsiae had slightly fewer expression of both enzymes ( $p f l$ median $=0.26 \% ; 25$ th $-75^{\text {th }}=0.2-0.6 \%$ and $l d h$ median $=0.06 \% ; 25$ th $-75^{\text {th }}=0.04-0.12 \%$ ). The $p f l$ was higher expressed than acetate kinase (acetate as the final end-product of the, Wilcoxon Rank Sum test; S. inopinata $\mathrm{p}=0.013$ and S. wiggsiae $\mathrm{p}=0.029$ ) and acetaldehyde-CoA/alcohol dehydrogenase (only present at $S$. wiggsiae) in both species (Wilcoxon
Rank Sum test; $S$. inopinata $\mathrm{p}=0.010$ and $S$. wiggsiae $\mathrm{p}=0.029$ ).

The expression of carbohydrate-modifying enzymes was also observed. Gene expression of pullulanase by $S$. inopinata ranged from 0.2 to $0.5 \%$ (median $=0.3 \% ; 25$ th $-75^{\text {th }}=0.3 \%-0.4 \%$ ). Pullulanase expression by $S$. wiggsiae ranged from 0.07 to $1.36 \%$ (median $=0.3 ; 25$ th $\left.-75^{\text {th }}=0.2-0.9\right)$. This enzyme is a glycosyl hydrolase that is responsible for the adaptation of complex carbohydrates (pullulan is produced from starch). Other glycosyl hydrolases with high gene expression included SCIP_RS00040 (median $\left.=0.08 \% ; 25 \mathrm{th}-75^{\text {th }}=0.0-0.1 \%\right)$, SCIP_RS05970 (median $=0.02 \% ; 25$ th $-75^{\text {th }}=0.0-0.0 \%$ ), SCIP_RS04605 (median $=0.08 \% ; 25$ th $-75^{\text {th }}=0.0-0.1 \%$, from $S$. inopinata; and HMPREF9156_RS04060 (median $=0.20$; 25 th $-75^{\text {th }}=0.1-0.2 \%$, from S. wiggsiae.

In the top 20 most expressed genes of $S$. inopinata, were observed the ones that code peptide $A B C$ transporter, $\mathrm{ABC}$ transporter ATP-binding protein and sugar $\mathrm{ABC}$ transporter permease, while for $\mathrm{S}$. wiggsiae $\mathrm{ABC}$ transporter ATP-binding protein (the highest median of reads in the genome), multidrug $A B C$ transporter ATP-binding protein, $\mathrm{ABC}$ transporter, $\mathrm{ABC}$ transporter substrate-binding protein, sugar $\mathrm{ABC}$ transporter permease, and peptide $\mathrm{ABC}$ transporter ATP-binding protein featured in the list of most highly expressed genes. A gene that codes a collagen adhesion protein was also among the most expressed genes in both S. inopinata and S. wiggsiae.

\section{Discussion}

This study set out to observe the gene expression profile of Scardovia spp. in natural samples of root caries, and compare it with that of other wellestablished cariogenic microorganisms. The most obvious finding to emerge from this study is a great relative prevalence of metabolism of $S$. inopinata and S. wiggisiae in root caries biofilms where Lactobacillus spp. were also highly relatively prevalent. The research has also shown that genes with functions in some important metabolic pathways were highly expressed in root caries, such as the "bifid shunt" and starch metabolism. These findings contribute to our understanding of Scardovia cariogenic potential 
Gene expression profile of Scardovia spp. in the metatranscriptome of root caries

Table. Top 30 most expressed genes of Scardovia inopinata and Scardovia wiggsiae in root caries samples.

\begin{tabular}{|c|c|c|c|c|c|c|c|c|c|}
\hline \multicolumn{2}{|c|}{ Scardovia inopinata JCM 12537} & \multicolumn{8}{|c|}{ Proportion of reads within the total reads of the genome } \\
\hline Gene code & Protein & RC_7 & RC_8 & RC_A & RC_B & RC_D & RC_F & RC_G & RC_H \\
\hline SCIP_RS06240 & pyridine nucleotide-disulfide oxidoreductase & 0.12 & 0.50 & 0.09 & 0.29 & 71.57 & 4.03 & 16.60 & 10.80 \\
\hline SCIP_RS04050 & peptidase S8 & 0.24 & 2.97 & 4.82 & 0.56 & 0.43 & 1.63 & 3.38 & 0.79 \\
\hline SCIP_RS02840 & phosphoketolase & 0.13 & 1.88 & 0.91 & 0.21 & 0.07 & 2.83 & 1.05 & 0.75 \\
\hline SCIP_RS04235 & peptide $A B C$ transporter & 0.09 & 1.49 & 2.82 & 0.23 & 0.18 & 0.48 & 2.10 & 0.44 \\
\hline SCIP_RS05945 & $A B C$ transporter & 0.04 & 0.00 & 0.03 & 0.05 & 5.64 & 0.01 & 0.01 & 0.94 \\
\hline SCIP_RS00115 & sugar $A B C$ transporter ATP-binding protein & 0.08 & 0.79 & 0.65 & 0.10 & 0.05 & 3.94 & 0.43 & 0.16 \\
\hline SCIP_RS03380 & transaldolase & 0.06 & 2.38 & 0.34 & 0.08 & 0.04 & 2.34 & 0.65 & 0.26 \\
\hline SCIP_RS03385 & transketolase & 0.12 & 2.38 & 0.45 & 0.17 & 0.08 & 1.34 & 1.16 & 0.33 \\
\hline SCIP_RS00890 & elongation factor Tu & 0.06 & 2.97 & 0.44 & 0.08 & 0.02 & 1.30 & 0.62 & 0.42 \\
\hline SCIP_RS01370 & ACP S-malonyltransferase & 0.60 & 0.10 & 0.92 & 0.66 & 0.18 & 1.88 & 0.61 & 0.96 \\
\hline SCIP_RS03780 & formate acetyltransferase & 0.16 & 0.99 & 0.68 & 0.19 & 0.08 & 2.23 & 0.63 & 0.37 \\
\hline SCIP_RS02240 & $A B C$ transporter substrate-binding protein & 0.06 & 1.88 & 0.20 & 0.08 & 0.00 & 1.34 & 0.39 & 0.48 \\
\hline SCIP_RS00740 & ribonucleoside-triphosphate reductase & 0.16 & 1.98 & 0.42 & 0.18 & 0.02 & 0.38 & 0.55 & 0.73 \\
\hline SCIP_RS00795 & collagen adhesin & 0.20 & 0.30 & 0.58 & 0.23 & 0.09 & 1.28 & 1.21 & 0.43 \\
\hline SCIP_RS01120 & DNA-directed RNA polymerase subunit beta & 0.25 & 1.19 & 0.71 & 0.27 & 0.04 & 0.43 & 0.91 & 0.33 \\
\hline SCIP_RS04410 & glyceraldehyde-3-phosphate dehydrogenase & 0.07 & 0.40 & 0.37 & 0.10 & 0.05 & 2.16 & 0.49 & 0.22 \\
\hline SCIP_RS01830 & $\begin{array}{c}\text { type I site-specific deoxyribonuclease } \\
\text { restriction subunit }\end{array}$ & 0.18 & 0.00 & 0.00 & 0.00 & 3.07 & 0.05 & 0.02 & 0.45 \\
\hline dnaK_74 & dnaK & 0.18 & 0.20 & 0.21 & 0.14 & 0.00 & 0.26 & 2.56 & 0.14 \\
\hline SCIP_RS01675 & ATPase AAA & 0.24 & 0.00 & 0.47 & 0.23 & 0.05 & 1.43 & 1.08 & 0.12 \\
\hline fusA_113 & elongation factor $\mathrm{G}$ fus $\mathrm{A}$ & 0.11 & 1.68 & 0.43 & 0.12 & 0.02 & 0.15 & 0.54 & 0.35 \\
\hline SCIP_RS00770 & hypothetical protein & 0.16 & 0.69 & 0.27 & 0.15 & 0.02 & 0.61 & 1.03 & 0.17 \\
\hline SCIP_RSO1125 & DNA-directed RNA polymerase subunit beta $\backslash^{\prime}$ & 0.28 & 0.40 & 0.77 & 0.28 & 0.04 & 0.54 & 0.37 & 0.42 \\
\hline SCIP_RS06520 & molecular chaperone Hspl 8 & 0.04 & 0.69 & 0.19 & 0.06 & 0.01 & 0.95 & 0.94 & 0.05 \\
\hline SCIP_RS05935 & hypothetical protein & 0.09 & 0.00 & 0.11 & 0.10 & 0.00 & 0.01 & 0.01 & 2.59 \\
\hline SCIP_RS06420 & hypothetical protein & 0.06 & 0.00 & 0.00 & 0.03 & 0.08 & 0.06 & 0.00 & 2.53 \\
\hline SCIP_RS05960 & pyridine nucleotide-disulfide oxidoreductase & 0.09 & 0.00 & 0.09 & 0.10 & 1.76 & 0.04 & 0.10 & 0.52 \\
\hline SCIP_RS04220 & peptide $A B C$ transporter ATP-binding protein & 0.13 & 1.78 & 0.15 & 0.15 & 0.06 & 0.09 & 0.12 & 0.13 \\
\hline SCIP_RS01555 & cell division protein DivIVA & 0.09 & 1.19 & 0.28 & 0.09 & 0.05 & 0.15 & 0.59 & 0.11 \\
\hline SCIP_RS02775 & pyruvate kinase & 0.08 & 0.69 & 0.13 & 0.10 & 0.00 & 1.06 & 0.30 & 0.13 \\
\hline SCIP_RS07225 & glycogen phosphorylase & 0.21 & 0.59 & 0.24 & 0.23 & 0.01 & 0.32 & 0.49 & 0.42 \\
\hline \multicolumn{2}{|l|}{ Scardovia wiggsiae F0424 } & \multicolumn{8}{|c|}{ Proportion of reads within the total reads of the genome } \\
\hline Gene code & Protein & RC_7 & & RC_A & RC_B & RC_D & RC_F & RC_G & $\mathrm{RC} \_\mathrm{H}$ \\
\hline HMPREF9156_RS06255 & $A B C$ transporter & 0.38 & & 7.48 & 0.54 & 0.20 & 0.30 & 0.06 & 0.04 \\
\hline HMPREF9156_RS06185 & $A B C$ transporter ATP-binding protein & 7.10 & & 0.05 & 0.17 & 0.04 & 0.15 & 0.32 & 0.15 \\
\hline HMPREF9156_RS06190 & multidrug $A B C$ transporter ATP-binding protein & 7.19 & & 0.11 & 0.14 & 0.02 & 0.15 & 0.22 & 0.15 \\
\hline HMPREF9156_RS06230 & pullulanase & 7.01 & & 0.04 & 0.14 & 0.02 & 0.00 & 0.27 & 0.15 \\
\hline HMPREF9156_RS02600 & chaperone protein $\mathrm{ClpB}$ & 0.08 & & 0.23 & 0.18 & 4.28 & 0.23 & 0.41 & 0.18 \\
\hline HMPREF9156_RSO2030 & $A B C$ transporter substrate-binding protein & 0.06 & & 0.34 & 0.10 & 2.95 & 1.44 & 0.43 & 0.19 \\
\hline HMPREF9156_RS06215 & hypothetical protein & 4.80 & & 0.08 & 0.06 & 0.02 & 0.08 & 0.11 & 0.09 \\
\hline
\end{tabular}

Continue 


\begin{tabular}{|c|c|c|c|c|c|c|c|c|}
\hline \multicolumn{9}{|l|}{ Continuation } \\
\hline HMPREF9156_RS06195 & endo-1,4-beta-xylanase & 4.47 & 0.13 & 0.12 & 0.01 & 0.00 & 0.24 & 0.09 \\
\hline HMPREF9156_RS02910 & ACP S-malonyltransferase & 0.27 & 0.66 & 0.75 & 0.87 & 0.68 & 0.92 & 0.67 \\
\hline HMPREF9156_RS06220 & septum formation protein Maf & 4.06 & 0.16 & 0.08 & 0.03 & 0.08 & 0.22 & 0.09 \\
\hline dnaK_73 & dnaK & 0.06 & 0.27 & 0.14 & 3.07 & 0.00 & 0.59 & 0.13 \\
\hline HMPREF9156_RS01510 & phosphoketolase & 0.07 & 0.28 & 0.24 & 1.36 & 1.59 & 0.45 & 0.24 \\
\hline HMPREF9156_RS04005 & sugar $A B C$ transporter ATP-binding protein & 0.01 & 0.17 & 0.09 & 2.66 & 0.84 & 0.35 & 0.11 \\
\hline HMPREF9156_RS00765 & formate acetyltransferase & 0.12 & 0.25 & 0.20 & 1.94 & 0.99 & 0.46 & 0.27 \\
\hline HMPREF9156_RS06235 & hypothetical protein & 3.79 & 0.03 & 0.11 & 0.01 & 0.00 & 0.18 & 0.11 \\
\hline HMPREF9156_RS03785 & peptidase S8 & 0.21 & 0.68 & 0.40 & 1.35 & 0.46 & 0.60 & 0.44 \\
\hline HMPREF9156_RS06180 & hypothetical protein & 3.53 & 0.08 & 0.09 & 0.02 & 0.00 & 0.19 & 0.10 \\
\hline HMPREF9156_RS02025 & sugar $A B C$ transporter permease & 0.12 & 0.28 & 0.11 & 1.10 & 1.82 & 0.27 & 0.16 \\
\hline HMPREF9156_RS06210 & homoserine dehydrogenase & 3.18 & 0.05 & 0.07 & 0.03 & 0.15 & 0.14 & 0.07 \\
\hline HMPREF9156_RS03140 & pullulanase & 0.18 & 0.53 & 0.40 & 0.83 & 0.91 & 0.40 & 0.39 \\
\hline HMPREF9156_RS06205 & $\begin{array}{c}\text { 2-hydroxyhepta-2,4-diene-1,7-dioate } \\
\text { isomerase }\end{array}$ & 3.17 & 0.07 & 0.08 & 0.02 & 0.00 & 0.05 & 0.06 \\
\hline HMPREF9156_RS06270 & hypothetical protein & 0.07 & 0.25 & 0.10 & 0.84 & 1.59 & 0.25 & 0.18 \\
\hline HMPREF9156_RS04820 & hypothetical protein & 0.13 & 0.41 & 0.35 & 0.74 & 0.53 & 0.61 & 0.32 \\
\hline HMPREF9156_RS03425 & elongation factor Tu & 0.06 & 0.29 & 0.08 & 0.86 & 1.21 & 0.34 & 0.15 \\
\hline HMPREF9156_RS00310 & glyceraldehyde-3-phosphate dehydrogenase & 0.09 & 0.07 & 0.10 & 1.28 & 0.99 & 0.31 & 0.10 \\
\hline HMPREF9156_RS05500 & $\begin{array}{l}\text { ATP-dependent Clp protease ATP-binding } \\
\text { subunit ClpC }\end{array}$ & 0.08 & 0.25 & 0.16 & 1.77 & 0.15 & 0.31 & 0.19 \\
\hline HMPREF9156_RS04550 & glycogen phosphorylase & 0.15 & 0.16 & 0.29 & 0.44 & 1.29 & 0.26 & 0.26 \\
\hline rpsN_43 & $\operatorname{rps} N$ & 0.34 & 0.05 & 0.01 & 0.05 & 2.35 & 0.02 & 0.01 \\
\hline HMPREF9156_RS06200 & YggS family pyridoxal phosphate enzyme & 2.54 & 0.03 & 0.06 & 0.01 & 0.00 & 0.14 & 0.05 \\
\hline HMPREF9156_RS03180 & DNA-directed RNA polymerase subunit beta $\backslash^{\prime}$ & 0.06 & 0.27 & 0.28 & 0.84 & 0.53 & 0.49 & 0.26 \\
\hline
\end{tabular}

and provide a basis for new investigations of their role in dysbiosis.

Many species have been found in root caries lesions, but studies are still focusing on wellestablished cariogenic microorganisms, such as S. mutans and Lactobacillus spp. However, previous studies reported low or highly variable percentages of these predicted cariogenic species in root caries ${ }^{16,21,22}$. Although culture and DNA-based studies showed low abundance of Lactobacillus sp. and S. mutans, the RNA-seq showed great gene expression here. Such approaches have also failed to address Scardovia spp. prevalence. In this study, the proportion of each, S. inopinata and S. wiggisae, reached approximately $6 \%$ of the total metatranscriptome of root caries. Scardovia has been detected in caries lesions, ${ }^{12,13,14}$ in association with root caries progression. ${ }^{9,10,16}$ It was defined as one of the organisms that could be a 'keystone' for coronal caries. ${ }^{15}$ It is interesting to note that only one sample had no expression at all of both species, and all other samples in the present study had activity of at least one species of Scardovia. In this sample, a great gene expression of Lactobacillus spp. and S. mutans was observed. We also sequenced biofilm samples from sound root surfaces; however, using the cut-off, only one out of 10 samples presented Scardovia (data not shown), suggesting that its prevalence is related to the root caries environment.

The correlation of the proportion of Scardovia within the metatranscriptome was evaluated and a significant positive correlation with Lactobacillus and Bifidobacteriaceae members was observed, while a non-significant negative correlation with $S$. mutans was found. Nyvad et al. ${ }^{23}$ characterized the root caries microbiota in vivo, suggesting that a dominance of 
a single or few microorganisms may reflect a very high cariogenicity. This is in line with the results of our study, which showed a predominance of one or few species, and a pattern of predominance of Lactobacillus spp. with others, but $S$. mutans appeared to be active as the main cariogenic species in the community. Eriksson et al..$^{24}$ suggested that in subjects with caries experience, high levels of $S$. mutans were associated with the presence of a few saccharolytic species, including $S$. wiggsiae. Similarly, a culturebased study showed that the presence of both $S$. wiggsiae and $S$. mutans was associated with severe early childhood caries. ${ }^{13}$ Another previous in vitro study showed that Scardovia acid production was higher when in association with S. mutans. ${ }^{11}$ However, our results showed that clinically this association is not preferable from both species regarding their metabolism in root caries.

Bifidobacteria possess a high number of enzymes involved in sugar metabolism. ${ }^{25}$ Phosphoketolase (Figure 5) is the key enzyme for the "bifid shunt" and both species of Scardovia possess it. This pathway allows the production of more energy from carbohydrates compared with that produced by the Embden-Meyerhof-Parnas pathway. ${ }^{26}$ Phosphoketolase is also a taxonomic marker for the family of Bifidobacteriaceae. Our results showed that this enzyme was the highest expressed in the OPP pathway enzymes in both $S$. inopinata and $S$. wiggsiae (Figure 5).

In general, high lactate production correlated with low amounts of acetate, ethanol and formate production and vice versa, ${ }^{27}$ and it is caused by the rapid consumption of an energy source. ${ }^{18,26}$ We observed high gene expression of lactate dehydrogenase $(l d h)$ and pyruvate formate lyase $(p f l)$ in Scardovia. Furthermore, the $p f l$ was significantly higher expressed than acetate kinase (in both species) and acetaldehyde-CoA/ alcohol dehydrogenase (only at $S$. wiggsiae), which could result in a higher concentration of lactate than acetate and alcohol production by these species, and this may be related to root caries progress.

The expression of carbohydrate-modifying enzymes was also observed. As all samples presented an expression of at least $0.2 \%$ of the whole transcriptome of the species, it suggests that this is an essential gene to survive in vivo in root caries. Pullulan is a polysaccharide polymer consisting of maltotriose units, and it is produced from starch and, thus, Scardovia may be metabolizing starch that is cariogenic to root caries. ${ }^{28}$

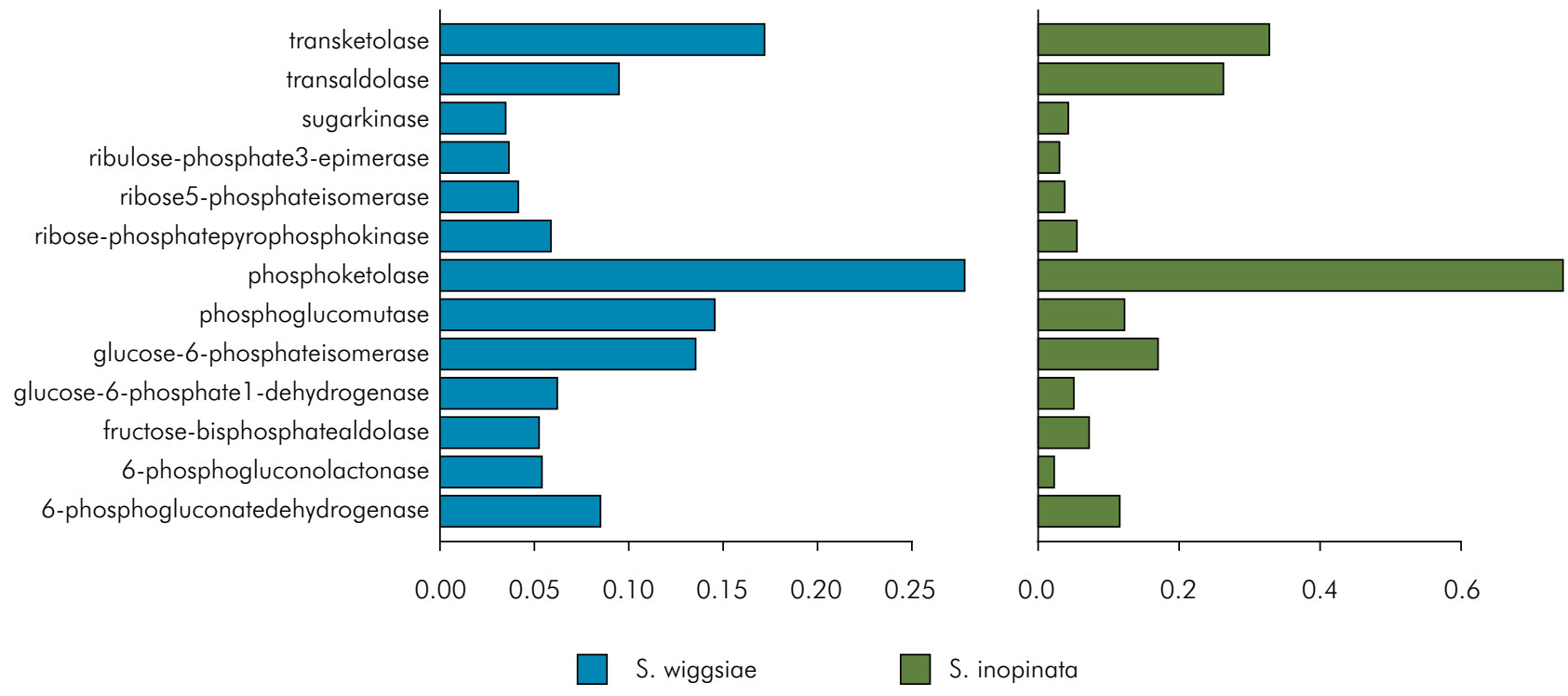

Figure 5. Proportion of reads assigned to genes that code Oxidative Pentose Phosphate Pathway enzymes in the transcriptome of Scardovia species. Median of gene expression at $n=8$ samples with putative presence of Scardovia. 
The literature has shown that the gene content of a bifidobacterial genome seems to reflect its adaptation, as indicated by the presence of genes that encode a variety of carbohydrate-modifying enzymes. As expected, many genes coding $\mathrm{ABC}$ transporters had high levels of expression in both species. ABC systems are responsible for the transport of nutrients such as mannose-containing oligosaccharides, while glucose is internalized using a glucose-specific PEP:PTS. ${ }^{18,26}$ It could suggest that in root caries, Scardovia spp. use low glucose but high complexity carbohydrates because it expresses more $A B C$ transporters than PEP:PTS systems. In contrast to $S$. mutans, which possess 14 PEP:PTS systems, only a minority of the sugars utilized by bifidobacteria are internalized via PEP:PTS. ${ }^{18,26}$

Strengths of this study include the type of methodology used (RNA-seq). As the target molecule of this study was mRNA, the result represents the proportion of general metabolic activity. The metatranscriptome allows the investigation of a large number of genes; however, specific aspects of how some species collaborate to promote root caries progression, as showed here, would be unmanageable by showing the results of the metatranscriptome as a whole. This account must be approached with some caution because it was based on short read mapping and this could add some bias in analysing reads at the species-level. This led us to use two different analysis pipelines to try overcome this issue. The contigs with $\sim 500 \mathrm{bp}$ were assembled and mapped to species.

A number of conclusions can be drawn from the present descriptive study. There is a positive correlation between the total metabolism of Lactobacillus spp., other Bifidobacteriaceae and Scardovia sp. in root caries, which could suggest a symbiotic relationship between these species. The Bifid shunt and starch metabolism are expressed in S. inopinata and S. wiggsiae in root caries. The understanding of Scardovia sp. as a protagonist in root caries might provide meaningful information for the development of future strategies of diagnosis and treatment.

\section{Acknowledgement}

The authors are grateful to the volunteers for their biofilm donations. Financial support was provided by Leeds Teaching Hospitals Charitable Foundation (R\&D/PP/12011), the Dunhill Medical Trust (R245/0212), the Brazilian National Counsel of Technological and Scientific Development (CNPQ) (process no. 482504/2013-7), the Coordination for the Improvement of Higher Level Education (CAPES) (process no 18097-12-0), and the Rio Grande do Sul State Foundation for Research Support (FAPERGS) (process no. 001/2013PQG).

\section{References}

1. Bowden GH, Hamilton IR. Survival of oral bacteria. Crit Rev Oral Biol Med. 1998;9(1):54-85. https://doi.org/10.1177/10454411980090010401

2. Zaura E, Keijser BJ, Huse SM, Crielaard W. Defining the healthy "core microbiome" of oral microbial communities. BMC Microbiol. 2009 Dec;9(1):259. https://doi.org/10.1186/1471-2180-9-259

3. Marsh PD. Microbiology of dental plaque biofilms and their role in oral health and caries. Dent Clin North Am. 2010 Jul;54(3):441-54. https://doi.org/10.1016/i.cden.2010.03.002

4. Aas JA, Griffen AL, Dardis SR, Lee AM, Olsen I, Dewhirst FE, et al. Bacteria of dental caries in primary and permanent teeth in children and young adults. J Clin Microbiol. 2008 Apr;46(4):1407-17. https://doi.org/10.1128/JCM.01410-07

5. Emilson CG, Ravald N, Birkhed D. Effects of a 12-month prophylactic programme on selected oral bacterial populations on root surfaces with active and inactive carious lesions. Caries Res. 1993;27(3):195-200. https://doi.org/10.1159/000261541

6. Gross EL, Leys EJ, Gasparovich SR, Firestone ND, Schwartzbaum JA, Janies DA, et al. Bacterial 16S sequence analysis of severe caries in young permanent teeth. J Clin Microbiol. 2010 Nov;48(11):4121-8. https://doi.org/10.1128/JCM.01232-10

7. Henne K, Rheinberg A, Melzer-Krick B, Conrads G. Aciduric microbial taxa including Scardovia wiggsiae and Bifidobacterium spp. in caries and caries free subjects. Anaerobe. 2015;35(Pt A):60-65. https://doi.org/10.1016/i.anaerobe.2015.04.011

8. Modesto M, Biavati B, Mattarelli P. Occurrence of the family bifidobacteriaceae in human dental caries and plaque. Caries Res. 2006;40(3):271-6. https://doi.org/10.1159/000092237 
9. Mantzourani M, Fenlon M, Beighton D. Association between Bifidobacteriaceae and the clinical severity of root caries lesions. Oral Microbiol Immunol. 2009 Feb;24(1):32-7. https://doi.org/10.1111/i.1399-302X.2008.00470.x

10. Beighton D, Lynch E, Heath MR. A microbiological study of primary root-caries lesions with different treatment needs. J Dent Res. 1993 Mar;72(3):623-9. https://doi.org/10.1177/00220345930720031201

11. Matos BM, Brighenti FL, Do T, Beighton D, Koga-lto CY. Acidogenicity of dual-species biofilms of bifidobacteria and Streptococcus mutans. Clin Oral Investig. 2017 Jun;21(5):1769-76. https://doi.org/10.1007/s00784-016-1958-1

12. Kressirer CA, Smith DJ, King WF, Dobeck JM, Starr JR, Tanner AC. Scardovia wiggsiae and its potential role as a caries pathogen. J Oral Biosci. 2017 Aug;59(3):135-41. https://doi.org/10.1016/i.job.2017.05.002

13. Tanner AC, Mathney JM, Kent RL, Chalmers NI, Hughes CV, Loo CY, et al. Cultivable anaerobic microbiota of severe early childhood caries. J Clin Microbiol. 2011 Apr;49(4):1464-74. https://doi.org/10.1128/JCM.02427-10

14. Tanner AC, Kent RL Jr, Holgerson PL, Hughes CV, Loo CY, Kanasi E, et al. Microbiota of severe early childhood caries before and after therapy. J Dent Res. 2011 Nov;90(11):1298-305. https://doi.org/10.1177/0022034511421201

15. Zhou J, Jiang N, Wang S, Hu X, Jiao K, He X, et al. Exploration of human salivary microbiomes: insights into the novel characteristics of microbial community structure in caries and caries-free subjects. PLoS One. 2016 Jan;11(1):e0147039. https://doi.org/10.1371/journal.pone.0147039

16. Houte J, Lopman J, Kent R. The predominant cultivable flora of sound and carious human root surfaces. J Dent Res. 1994 Nov;73(11):1727-34. https://doi.org/10.1177/00220345940730110801

17. Valdez RM, Santos VR, Caiaffa KS, Danelon M, Arthur RA, Negrini TC, et al. Comparative in vitro investigation of the cariogenic potential of bifidobacteria. Arch Oral Biol. 2016 Nov;71:97-103. https://doi.org/10.1016/i.archoralbio.2016.07.005

18. Pokusaeva K, Fitzgerald GF, Sinderen D. Carbohydrate metabolism in Bifidobacteria. Genes Nutr. 2011 Aug;6(3):285-306. https://doi.org/10.1007/s12263-010-0206-6

19. Dame-Teixeira N, Parolo CC, Maltz M, Tugnait A, Devine D, Do T. Actinomyces spp. gene expression in root caries lesions. J Oral Microbiol. 2016 Sep;8(1):32383. https://doi.org/10.3402/jom.v8.32383

20. Fejerskov O, Luan WM, Nyvad B, Budtz-Jørgensen E, Holm-Pedersen P. Active and inactive root surface caries lesions in a selected group of 60- to 80-year-old Danes. Caries Res. 1991;25(5):385-91. https://doi.org/10.1159/000261396

21. Emilson CG, Klock B, Sanford CB. Microbial flora associated with presence of root surface caries in periodontally treated patients. Scand J Dent Res. 1988 Feb;96(1):40-9. https://doi.org/10.1111/j.1600-0722.1988.tb01406.x

22. Chen $L, Q i n B, D u M$, Zhong $H, X \cup Q$, Li Y, et al. Extensive description and comparison of human supra-gingival microbiome in root caries and health. PLoS One. 2015 Feb;10(2):e0117064. https://doi.org/10.1371/journal.pone.0117064

23. Nyvad B, Kilian M. Microflora associated with experimental root surface caries in humans. Infect Immun. 1990 Jun;58(6):1628-33. https://doi.org/10.1128/IAl.58.6.1628-1633.1990

24. Eriksson L, Lif Holgerson P, Esberg A, Johansson I. Microbial complexes and caries in 17-year-olds with and without Streptococcus mutans. J Dent Res. 2018 Mar;97(3):275-282. https://doi.org/10.1177/0022034517731758

25. Milani C, Lugli GA, Duranti S, Turroni F, Bottacini F, Mangifesta M, et al. Genomic encyclopedia of type strains of the genus Bifidobacterium. Appl Environ Microbiol. 2014 Oct;80(20):6290-302. https://doi.org/10.1128/AEM.02308-14

26. O'Callaghan A, Sinderen D. Bifidobacteria and Their Role as Members of the Human Gut Microbiota. Front Microbiol. 2016 Jun;7:925. https://doi.org/10.3389/fmicb.2016.00925

27. Palframan RJ, Gibson GR, Rastall RA. Carbohydrate preferences of Bifidobacterium species isolated from the human gut. Curr Issues Intest Microbiol. 2003 Sep;4(2):71-5.

28. Botelho JN, Villegas-Salinas M, Troncoso-Gajardo P, Giacaman RA, Cury JA. Enamel and dentine demineralization by a combination of starch and sucrose in a biofilm - caries model. Braz Oral Res. 2016 May;30(1):S1806-83242016000100250. https://doi.org/10.1590/1807-3107BOR-2016.vol30.0052 